\title{
ON MULTIVARIATE POWER SERIES OF RANDOM VARIABLES SATISFYING SOME HIERARCHY CONDITIONS
}

\section{Neuenschwander ${ }^{1}$}

Let $\left\{X_{n}\right\}_{n \geqslant 1}$ be a sequence of i.i.d. random vectors such that the distribution of $X_{n}$ is uniquely determined by its moments. We show that for a multivariate power series in the components of $\left\{X_{n}\right\}_{n} \geqslant 1$ that satisfies some algebraic "hierarchy condition" with respect to the vector components, whose coefficients fulfill a suitable positivity property, and which is absolutely $\mathcal{L}^{p}$-convergent for all $p \geqslant 1$, the distribution of the series determines uniquely the distribution of $X_{n}$. In statistical language, this means that the distribution (if it can be observed, e.g., by sampling from several i.i.d. models) of the power series under consideration is a sufficient statistic for the distribution of $X_{n}$. Special cases are generalized ARMA models where the dependence structure is not necessarily linear. This applies, e.g., to stock price models with random volatility and to mechanics (kinetic energy). The assertion can also be generalized to the case where the coefficients of the power series form a stochastic process that is independent of $\left\{X_{n}\right\}_{n \geqslant 1}$.

\section{Introduction and statement of results}

Throughout this paper, we will write $a=\left(a^{(1)}, a^{(2)}, \ldots, a^{(d)}\right)$ for a vector $a \in \mathbb{R}^{d}$ and its components.

Let $\left\{X_{n}\right\}_{n \geqslant 1}$ be a sequence of i.i.d. random vectors. In our result we will prove that the distribution of certain multivariate power series of the components of $\left\{X_{n}\right\}_{n} \geqslant 1$ are sufficient statistics for the distribution of $X_{n}$, provided the latter is uniquely determined by its moments. This means that if we can observe the distribution of the series (e.g., by sampling from i.i.d. models), then the distribution of the $X_{n}$ is uniquely determined. The method will be a recursive calculation of the moments of $X_{n}$, which can be done by imposing a certain "hierarchy" condition on the algebraic dependence of the vector components. That is why we will have to suppose that the distribution of $X_{n}$ is so-called determinate, i.e. uniquely determined by its moments. In precise formulation, this means that the distribution of $X_{n}$ is $\mathcal{L}^{k}$-integrable for all $k \in \mathbb{N}$ and that there is no other distribution which is $\mathcal{L}^{k}$-integrable for all $k \in \mathbb{N}$ and such that all moments (in the sense of "mixed moments" of the vector components) coincide with the corresponding moments of the distribution of $X_{n}$. Of course, distributions with analytic characteristic function (Fourier transform) are determinate. A well-known sufficient condition for determinacy of a distribution of a real-valued random variable $X$ is the so-called Carleman criterion: Write $m_{k}:=E\left(|X|^{k}\right)$. Then in the case

$$
\sum_{k=1}^{\infty} m_{2 k}^{-1 /(2 k)}=\infty
$$

the distribution of $X$ is determinate.

We will prove the following result:

Theorem 1. For fixed $h=1,2$, let $\left\{X_{h, n}\right\}_{n \geqslant 1}$ be sequences of i.i.d. $\mathbb{R}^{d}$-valued random vectors such that the distribution of $X_{1, n}$ is determinate. Assume $Z_{h}$ are $\mathbb{R}^{d}$-valued vectors with the property that every component $Z_{h}^{(i)}, 1 \leqslant i \leqslant d$, is a power series with random entries from $\left\{X_{h, n}^{(j)}\right\}_{n \geqslant 1 ; 1 \leqslant j \leqslant d}$ that converges absolutely in $\mathcal{L}^{p}$ for all $p \geqslant 1$ and which is of the form

$$
Z_{h}^{(i)}=L^{[i]}+P^{[i]},
$$

where $L^{[i]}$ is a linear form in the random variables $\left\{X_{h, n}^{(i)}\right\}_{n \geqslant 1}$ with coefficients $\left\{\gamma_{n}^{[i]}\right\}_{n \geqslant 1} \subset[0, \infty[$ (independent of $h$ ) and where $P^{[i]}$ is a power series in the random variables $\left\{X_{h, n}^{(j)}\right\}_{n \geqslant 1 ; 1 \leqslant j \leqslant i-1}$ and whose coefficients are also independent of $h$. Assume that there exists an $n \geqslant 1$ such that $\gamma_{n}^{[i]}>0$ for all $i \in\{1,2, \ldots, d\}$. Suppose furthermore that the distribution of $Z_{1}$ coincides with that of $Z_{2}$. Then also the distributions of $X_{1, n}$ and $X_{2, n}$ have to coincide.

\footnotetext{
${ }^{1}$ Université de Lausanne, Ecole des Hautes Etudes Commerciales, Institut de Sciences Actuarielles, CH-1015 Lausanne, and Universität Bern, Insitut für mathematische Statistik und Versicherungslehre, CH-3012 Bern, Switzerland. E-mail: daniel.neuenschwander@bluewin.ch
}

Proceedings of the XXVII International Seminar on Stability Problems for Stochastic Models, Nahariya, Israel, 22-26 October 2007. 
Applications are, e.g., generalized ARMA models in the sense that the dependence structure is not necessarily linear but possibly of higher degree and obeying an algebraic hierarchy structure with respect to the vector components to meet the conditions of Theorem 1. Let $\left\{Y_{n}\right\}_{n \in \mathbb{Z}}$ be an $\operatorname{ARMA}(k, \ell)$ model. That means that there are coefficients $\alpha_{i}, 1 \leqslant i \leqslant k$, and $\beta_{j}, 1 \leqslant j \leqslant \ell$, such that

$$
Y_{n}=\sum_{i=1}^{k} \alpha_{i} Y_{n-k-1+i}+\sum_{j=1}^{\ell} \beta_{j} T_{n-\ell+j}, \quad n \in \mathbb{Z},
$$

where $\left\{T_{n}\right\}_{n \in \mathbb{Z}}$ is a sequence of i.i.d. random variables. It is well known that under certain conditions, this allows for a representation

$$
Y_{n}=\sum_{-\infty<m \leqslant n} \gamma_{m} T_{m}
$$

for suitable coefficients $\left\{\gamma_{n}\right\}_{n \in \mathbb{Z}}$. This corresponds to the special case $d=1$ in our model (with the obvious settings $X_{m}:=T_{n+1-m}$ and $\left.\gamma_{m}^{[1]}:=\gamma_{n+1-m}, m \geqslant 1\right)$. As a generalization, one could now, e.g., imagine multivariate time series models of the form

$$
Y_{n}^{(i)}=H_{n}^{[i]}+Q_{n}^{[i]},
$$

where $H_{n}^{[i]}$ is a linear form in the random variables $\left\{T_{m}^{(i)}\right\}_{-\infty<m \leqslant n}$ and $Q_{n}^{[i]}$ is a power series in the random variables $\left\{T_{m}^{(j)}\right\}_{-\infty<m \leqslant n-1 ; 1 \leqslant j \leqslant i-1}$. Here, of course, by analogy to the one-dimensional situation, $\left\{T_{n}\right\}_{n \in \mathbb{Z}}$ are i.i.d. $d$-dimensional random vectors. If, for example, $d=3, Q_{n}^{[i]}=0, i=1,2, L^{[3]}=0$, and

$$
Q_{n}^{[3]}=\sum_{-\infty<m \leqslant n}\left(\sum_{-\infty<\ell \leqslant m-1} \gamma_{\ell}^{[1]} T_{\ell}^{(1)}\right) \gamma_{m}^{[2]} T_{m}^{(2)},
$$

then one can, for $n \in \mathbb{Z}$, e.g. interpret $\gamma_{n}^{[2]} T_{n}^{(2)}$ as the length of the displacement of a particle between times $n-1$ and $n$ and $\gamma_{n}^{[1]} T_{n}^{(1)}$ as the increment of the force acting on this particle. In this case, $Y_{n}^{(3)}$ is the kinetic energy at time $n \in \mathbb{Z}$. That means that (under the assumptions of Theorem 1) if we have two mechanical experiments where the joint distributions of the place, the force, and the kinetic energy at some fixed time point can be observed and are found to coincide, then in both experimental situations the joint distributions of the displacement and the force process have to be equal. Another application of this model, as we have just considered it for mechanics, is, e.g., in mathematical finance in connection with stock price models with stochastic volatility. Such models have been considered, e.g., in $[1,3,4]$. Here $\left\{Y_{n}^{(2)}\right\}_{n \in \mathbb{Z}}$ represents some "ground process" whose increments have deterministic variance and $\left\{Y_{n}^{(1)}\right\}_{n \in \mathbb{Z}}$ provides some random volatility. The "combined" process $\left\{Y_{n}^{(3)}\right\}_{n \in \mathbb{Z}}$ can then be used as a model for the logarithm of a stock price process with random volatility. Then also here (by analogy to the situation considered in [4]) in the case where the ground process and the stochastic volatility are assumed to be observable data themselves at least at some fixed time point $N$ (e.g., if they are also traded assets themselves), the joint distribution of all these three available market data (i.e., the ground process, the volatility itself, and the process stemming from the ground process by imposing the stochastic vvolatility as described) available at time point $N$ is a sufficient statistic for the distribution of the whole model (under the assumptions of Theorem 1).

\section{Proof of the result}

Now we come to the proof of Theorem 1.

Proof of Theorem 1. The principal method will be a recursive calculation of moments in the same spirit as it was used, e.g., in $[4,5]$.

Consider on $\mathbb{N}_{0}^{d}$ the lexicographic ordering from behind defined as follows: Put $\left(a_{1}, a_{2}, \ldots, a_{d}\right)<\left(b_{1}, b_{2}, \ldots, b_{d}\right)$ if $\left(a_{d}, a_{d-1}, \ldots, a_{d-j+1}\right)=\left(b_{d}, b_{d-1}, \ldots, b_{d-j+1}\right)$ and if $a_{d-j}<b_{d-j}$ for some $j \in\{0,1, \ldots, d-1\}$. By the absolute convergence, we may, for $h=1,2$ and $\ell=\left(\ell_{1}, \ell_{2}, \ldots, \ell_{d}\right) \in \mathbb{N}_{0}^{d}$, define the "mixed moments"

$$
\mu_{h, \ell}:=E\left(\prod_{j=1}^{d}\left(Z_{h}^{(j)}\right)^{\ell_{j}}\right)
$$


and

$$
m_{h, \ell}:=E\left(\prod_{j=1}^{d}\left(X_{h, n}^{(j)}\right)^{\ell_{j}}\right) .
$$

By our hypothesis in the theorem, we have that $\mu_{1, \ell}=\mu_{2, \ell}$ for all $\ell \in \mathbb{N}_{0}^{d}$. Now we will calculate, for $h=1,2$, the $m_{h, \ell}$ from the $\left\{\mu_{h, \ell}\right\}_{\ell \in \mathbb{N}_{0}^{d}}$ recursively with respect to the lexicographic ordering from behind of $\ell \in \mathbb{N}_{0}^{d}$; the determinacy of the distribution of $X_{1, n}$ assumed in the theorem will then yield the assertion to be verified. First, recall that

$$
\begin{aligned}
\mu_{h, \ell} & =E\left(\prod_{j=1}^{d}\left(Z_{h}^{(j)}\right)^{\ell_{j}}\right)= \\
& =E\left(\prod_{j=1}^{d}\left(L^{[j]}\left(\left\{X_{h, n}^{(j)}\right\}_{n \geqslant 1}\right)+P^{[j]}\left(\left\{X_{h, n}^{(i)}\right\}_{n \geqslant 1 ; 1 \leqslant i \leqslant j-1}\right)\right)^{\ell_{j}}\right) .
\end{aligned}
$$

One gets the relation

$$
\left(Z_{h}^{(j)}\right)^{\ell_{j}}=\sum_{n=1}^{\infty}\left(\gamma_{n}^{[j]}\right)^{\ell_{j}}\left(X_{h, n}^{(j)}\right)^{\ell_{j}}+V^{[j]}
$$

where $V^{[j]}$ is a power series in the variables $\left\{X_{h, n}^{(i)}\right\}_{n \geqslant 1 ; 1 \leqslant i \leqslant j}$ where in every monomial of $V^{[j]}$ the exponent of $X_{h, n}^{(j)}$ is strictly smaller than $\ell_{j}$. Now, by multiplying out, we obtain

$$
\begin{aligned}
\mu_{h, \ell} & =E\left(\prod_{j=1}^{d}(\ldots)^{\ell_{j}}\right)= \\
& =\sum_{n=1}^{\infty} \prod_{j=1}^{d}\left(\gamma_{n}^{[j]}\right)^{\ell_{j}} \cdot E\left(\prod_{j=1}^{d}\left(X_{h, n}^{(j)}\right)^{\ell_{j}}\right)+E(W)= \\
& =\sum_{n=1}^{\infty} \prod_{j=1}^{d}\left(\gamma_{n}^{[j]}\right)^{\ell_{j}} \cdot m_{n, \ell}+E(W),
\end{aligned}
$$

where $W$ is a power series in the variables $\left\{X_{h, n}^{(j)}\right\}_{n \geqslant 1 ; 1 \leqslant j \leqslant d}$ with the property that for every monomial

$$
\prod_{j=1}^{d}\left(X_{h, n}^{(j)}\right)^{r_{n, j}}
$$

we have $\left(r_{n, 1}, r_{n, 2}, \ldots, r_{n, d}\right)<\ell, n \geqslant 1$. So by the assumptions on the coefficients $\left\{\gamma_{n}^{[j]}\right\}_{n \geqslant 1 ; 1 \leqslant j \leqslant d}$ made in Theorem 1 and the induction hypothesis, Eq. (4) can be resolved uniquely with respect to the $m_{h, \ell}, \ell \in \mathbb{N}_{0}^{d}$, which entails that they must, for every fixed $\ell \in \mathbb{N}_{0}^{d}$, be equal for $h=1,2$. This finishes the proof.

\section{A generalization}

It is obvious from the above proof that the assertion of Theorem 1 remains true if $\left\{\gamma_{n}\right\}_{n \geqslant 1}\left(\gamma_{n}=\left\{\gamma_{n}^{[j]}\right\}_{1 \leqslant j \leqslant d}\right)$ is assumed to be a stochastic process with values in $\left[0, \infty{ }^{d}\right.$ which is independent of the $\left\{X_{h, n}\right\}_{n \geqslant 1}$ and such that for at least one $n \geqslant 1$, we have that with positive probability all components $\gamma_{n}^{[j]}, j=1,2, \ldots, d$, are positive. In this situation, the above proof can be generalized by just replacing the

$$
\prod_{j=1}^{d}\left(\gamma_{n}^{[j]}\right)^{\ell_{j}}
$$

by their expectations. 


\section{REFERENCES}

1. J. Hull and A. White, "The pricing of options on assets with stochastic volatility," J. Finance, XLII, No. 2, 281-300 (1987).

2. D. Neuenschwander, "On the uniqueness problem for continuous convolution semigroups of probability measures on simply connected nilpotent Lie groups," Publ. Math. Debrecen, 53, No. 3-4, 415-422 (1998).

3. D. Neuenschwander, "On option pricing in models driven by iterated integrals of Brownian motions," Mitt. $S A V, \mathbf{1}, 35-39$ (2000).

4. D. Neuenschwander, "Retrieval of Black-Scholes and generalized Erlang models by perturbed observations at a fixed time," Insur. Math. Econ., 42, No. 1, 453-458 (2008).

5. D. Neuenschwander, "Uniqueness of embedding of gaussian probability measures into a continuous convolution semigroup on simply connected nilpotent Lie groups," C. R., Math., Acad. Sci. Paris, 346, No. 15-16, 887-892 (2008). 